\title{
A Simple Sliding Mode Control for Saucer-shaped Aircraft
}

\author{
Lingling Wang ${ }^{\mathrm{a}}$, Huali $\mathrm{Wu}^{\mathrm{b}}$, Lili Yang, Junwei Lei \\ Department of Control, Naval Aeronautical and Astronautical University, Yantai, 264001, China \\ aemail:lingling0711@163.com, bemail:tom_linhuali@126.com
}

Keywords: Sliding Mode Control; Saucer-shaped Aircraft, Stability, Lyapunov function

\begin{abstract}
In this paper, a simple sliding mode control scheme, in which only the angular velocity and attitude angle have to be measured, is proposed to solve the non-minimum phase problem for a class of simplified linear model of saucer-shaped aircraft. The boundary of all closed-loop signals of the system is guaranteed by the selection and proof of the Lyapunov energy function. Finally, the simulation results show that the proposed method is correct and the control algorithm is simple and convenient.
\end{abstract}

\section{Introduction}

When the conventional aircraft is flying, its course control is realized by the deflection control of the rudder. But for the saucer-shaped aircraft, which is a disk shape without a tail or rudder, it is a kind of unconventional aircraft. Due to the full integration of the wing with the body, it is impossible to complete the flight control with the traditional rudder control method [1-2]. At present, most scholars use the combination of variable mass moment control and thrust vector control to achieve flight control [1-2]. Variable mass moment control is to change the control torque by changing the center of mass of the aircraft. The thrust vector control is to change the angle of the nozzle, and thus control the torque by utilizing the side directional thrust from the motor. But this composite control method has a series of problems to be solved, such as how to deal with the highly nonlinear system with multi inputs, and how to coordinate the two actuators between moving mass control and thrust vector control [3-6]. From the point of model design, the longitudinal dynamic model of saucer-shaped aircraft has been simplified in this paper, and then the sliding mode control method is used to control the angular velocity and attitude angle of aircraft for controller design. The effectiveness of this control algorithm is verified by simulation results.

\section{Model Description}

According to literature [7], the dynamic model for saucer-shaped aircraft can be described as formula 1 .

$$
\left\{\begin{array}{l}
M \dot{v}=-X-M g \sin \theta+P \cos (\xi+\alpha) \\
M v \dot{\theta}=Y-M g \cos \theta+P \sin (\xi+\alpha) \\
J_{z}^{\prime} \dot{\omega}_{d b z}^{b}=X_{P} P \sin \xi+y_{T} P \cos \xi+M_{z}+M_{z}\left(x_{b}\right)-\mu P \sin \xi \cdot x_{b} \\
\dot{\vartheta}=\omega_{d b z}^{b}
\end{array}\right.
$$

Theorem1: Assume the linear approximation system of nonlinear system is asymptotically stable, and then any linear feedback that can make the linear approximation system stable can also asymptotically stabilize the original nonlinear system, and at least make the nonlinear system locally stable [5].

The linear dynamic model of missile is studied in literature [7]. Similarly, we can also study the linear approximation system of nonlinear system as described in formula 1 , in order to find the control law which can make the original nonlinear system asymptotically stable. According to the literature [7-10], the linear approximation system for the system as described in formula 1 can be presented in formula 2. 


$$
\left\{\begin{array}{l}
\dot{\omega}_{z}=-a_{24} \theta+a_{24} \vartheta+a_{25} x_{b}+a_{z}^{\prime} \xi \\
\dot{\theta}=\left(a_{33}-a_{34}\right) \theta+a_{34} \vartheta+a_{x} \xi \\
\dot{\vartheta}=\omega_{z}
\end{array}\right.
$$

The definitions for all the symbols in formula 2 can be found in reference [7].

The transfer function can be derived from the above state equation, and this is a non-minimum phase system, as described in formula 3 , where $a_{z}<0, a_{34}>0, a_{24}>0, a_{x}>0$.

$$
G_{\xi}^{\vartheta}=\frac{a_{z}\left(S+a_{34}\right)-a_{x} a_{24}}{S\left[S^{2}+a_{34} S-a_{24}\right]}
$$

\section{Simple Sliding Mode Controller Design}

Design a simple sliding curve as in literature [8-10]:

$$
\sigma=c_{1}\left(\vartheta-\vartheta^{*}\right)+\omega_{z}
$$

Get the derivative as

$$
\dot{\sigma}=c_{1} \omega_{z}+\dot{\omega}_{z}
$$

Then it holds:

$$
\dot{\sigma}=c_{1} \omega_{z}+\left(-a_{24} \theta+a_{24} \vartheta+a_{25} x_{b}+a_{z}^{\prime} \xi\right)
$$

Choose an auxiliary control variable as

$$
u=a_{25} x_{b}+a_{z}^{\prime} \xi
$$

Then design the sliding control law as the formula 4 .

$$
u=-c_{1} \omega_{z}-\left(-a_{24} \theta+a_{24} \vartheta\right)-k_{1} \operatorname{sign}(\sigma)-k_{2} \frac{\sigma}{|\sigma|+\varepsilon}
$$

Then we can define a Lyapunov function as

$$
V=\frac{1}{2} \sigma^{2}
$$

And solve its derivative as

$$
\dot{V}=\sigma \dot{\sigma}
$$

Then it satisfies

$$
\dot{V}=-k_{1}|\sigma|-k_{2} \frac{\sigma^{2}}{|\sigma|+\varepsilon} \leq 0
$$

And according to Lyapunov theorem, it is easy to prove that the system is stable, so the design is reasonable.

\section{Simulation analysis}

To testify the rightness of the above simple backstepping control strategy, we use Matlab software to do the numerical simulation analysis. And the model parameter can see below table 1 , and We choose $\mathrm{c} 1=155, \mathrm{k} 1=0.05, \mathrm{k} 2=15, \mathrm{k} 3=5$, esten $=0.5$, and the simulation results are shown in figure 1 and figure 2 .

Table 1 Kinetic coefficients

\begin{tabular}{|c|c|c|c|c|c|}
\hline$a_{24}$ & $a_{25}$ & $a_{34}$ & $a_{37}$ & $a_{x}$ & $a_{z}\left(a_{z}^{\prime}\right)$ \\
\hline 829.0773 & -19.1620 & 2.3230 & 0.0013 & 0.0289 & -12.8979 \\
\hline
\end{tabular}




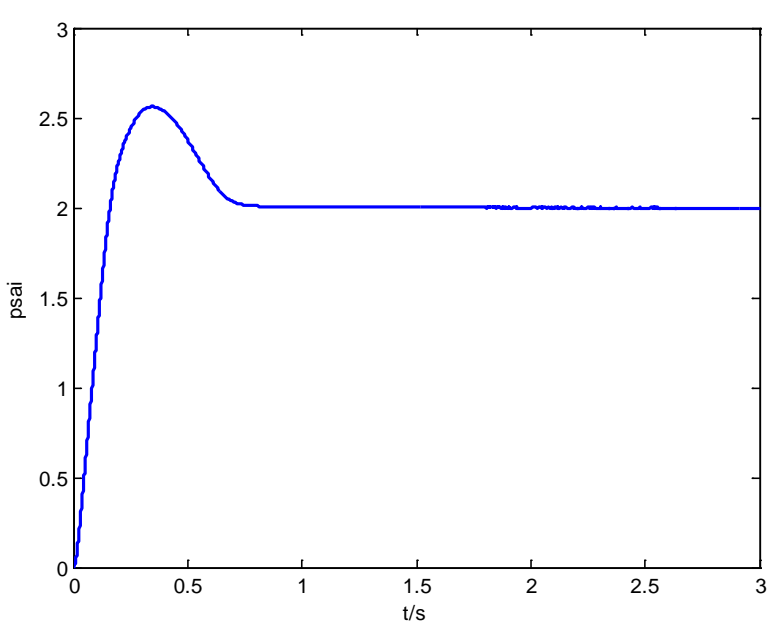

Figure 1 Curve of attitude angle of aircraft

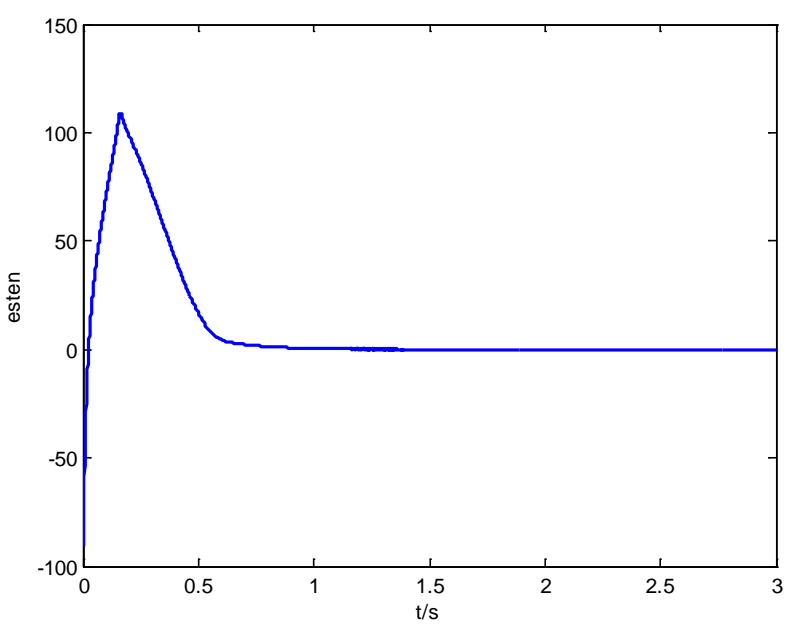

Figure 2 Curve of jet control

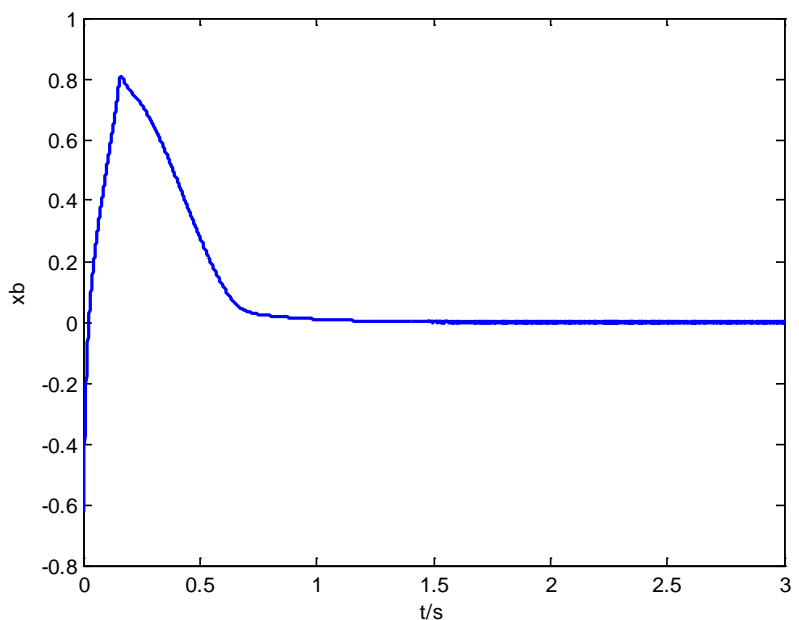

Figure 2 Curve of mass control

Figure 1 shows the attitude angle of aircraft is stable and the rise time is small than $0.1 \mathrm{~s}$. That is a very fast speed of saucer shaped aircraft. And figure 2 shows the jet control, figure 3 shows the mass control of $x_{b}$. So it means that we only used the Mass control and do not use the jet control.

\section{Conclusion}

In this paper, a sliding mode control scheme is designed to control the angular velocity and attitude 
angle of saucer-shaped aircraft from the simplified longitudinal dynamic function. The simulation results show that the sliding mode control algorithm can realize the longitudinal flight control of the saucer-shaped aircraft, and ensure the boundary of all closed-loop signals, thus solving the control problem of non-minimum phase.

\section{References}

[1] Gu Wenjin, Yang Kan, Research on GPC the saucer-like air vehicle by GPC [J]. Computer Simulation, 2011, 28(3):91-96(in Chinese).

[2] Peng Shan-guo, Yang Kan, Gu Wenjin, Research on compound control method of saucer-like air vehicle based on VSC[J]. 2010, 27(11):32-40(in Chinese).

[2] Yu Junli, Wang Linlin, Gao Ge, Using wing tip devices to improve performance of saucer-shaped aircraft [J]. Chinese Journal of Aeronautics, 2006, 19(4):309-314.

[4] Ma Songhui, Wu Chengfu, Chen Huaimin. Study on stability and manoeuvrability of flying wing aircraft [J]. Flight Dynamics, 2006, 24 (3):18-19(in Chinese).

[5] Wu Sentang, Fei Yuhua, Flight control systems [M]. Beijing: Beijing University of Aeronautics and Astronautics Press, 2005:73-88(in Chinese).

[6] Wang Jindong, Shen Xialing, GaoGe, Experimental research of low speed serodynamic characteristics of saucer-like lifting body[J]. Journal of Beijing University of Aeronautics and Astronautics, 2003,29(4):346-349(in Chinese).

[7] Wu Jinhuang, Xu Shenghong, Gu Wen-jin. Distribution Strategy based on Genetic-Fuzzy Logic for Compound Control of Flying Saucer [J]. Fire Control and Command Control, 2008, 33(5):63-65 (in Chinese).

[8] Wu Yinsheng, Shen qinglou, Shi Jianhong. Sliding Mode Control Based on Neural Network for Saucer-Shaped Aircraft with Saturation Compensation [J]. Journal of Naval Aeronautical and Astronautical University, 2012, 27(3):241-243 (in Chinese).

[9] K. Jamoussi, L. Chrifi-Alaoui, H. Benderradji, A. El Hajjaji, M. Ouali. Robust Sliding Mode Control Using Adaptive Switching Gain for Induction Motors [J]. International Journal of Automation and Computing, 2013, 10(4):303-311.

[10] LP Liu, ZM Fu, XN Song, Sliding Mode Control with Disturbance Observer for a Class of Nonlinear Systems [J]. International Journal of Automation \& Computing, 2012, 9(5):487-491. 